\title{
Antibody responses in furunculosis patients vaccinated with autologous formalin-killed Staphylococcus aureus
}

\author{
S. Holtfreter • J. Jursa-Kulesza • H. Masiuk • N. J. Verkaik • C. de Vogel • J. Kolata • \\ M. Nowosiad • L. Steil • W. van Wamel • A. van Belkum • U. Völker • \\ S. Giedrys-Kalemba • B. M. Bröker
}

Received: 5 December 2010 /Accepted: 17 December 2010/Published online: 22 April 2011

(C) The Author(s) 2011. This article is published with open access at Springerlink.com

\begin{abstract}
Autologous vaccines (short: autovaccines) have been used since the beginning of the 20th century to treat chronic staphylococcal infections, but their mechanisms of action are still obscure. This prospective pilot study involved four patients with furunculosis who were vaccinated with autologous formalin-killed Staphylococcus aureus cells. Vaccines were individually prepared from the infecting $S$. aureus strain and repeatedly injected subcutaneously in
\end{abstract}

Silva Holtfreter now works at the Department of Molecular Medicine and Pathology, University of Auckland, New Zealand.

Electronic supplementary material The online version of this article (doi:10.1007/s10096-010-1136-3) contains supplementary material, which is available to authorized users.

\author{
S. Holtfreter · J. Kolata · B. M. Bröker \\ Institute of Immunology and Transfusion Medicine, \\ University of Greifswald, \\ Greifswald, Germany
}

J. Jursa-Kulesza $\cdot$ H. Masiuk $\cdot$ M. Nowosiad $\cdot$ S. Giedrys-Kalemba Department of Microbiology and Immunology,

Pomeranian Medical University,

Szczecin, Poland

N. J. Verkaik · C. de Vogel · W. van Wamel · A. van Belkum Department of Medical Microbiology and Infectious Diseases, Erasmus Medical Center,

Rotterdam, The Netherlands

L. Steil · U. Völker

Interfaculty Institute of Genetics and Functional Genomics,

University of Greifswald,

Greifswald, Germany

S. Holtfreter $(\bowtie)$

Department of Molecular Medicine and Pathology,

University of Auckland,

85 Park Road, Grafton,

Auckland 1023, New Zealand

e-mail: s.holtfreter@auckland.co.nz increasing doses over several months. We characterized the virulence gene repertoire and spa genotype of the infecting and colonising $S$. aureus strains. Serum antibody responses to secreted and surface-bound bacterial antigens were determined by two-dimensional immunoblotting and flowcytometry based assays $\left(\right.$ Luminex $\left.{ }^{\circledR}\right)$. All patients reported clinical improvement. Molecular characterization showed that all strains isolated from one patient over time belonged to the same $S$. aureus clone. Already before treatment, there was robust antibody binding to a broad range of staphylococcal antigens. Autovaccination moderately boosted the IgG response to extracellular antigens in two patients, while the antibody response of the other two patients was not affected. Similarly, vaccination moderately enhanced the antibody response against some staphylococcal surface proteins, e.g. ClfA, ClfB, SdrD and SdrE. In summary, autovaccination only slightly boosted the pre-existing serum antibody response, predominantly to bacterial surface antigens.

\section{Introduction}

Besides being a common colonizer of human skin and mucosa, Staphylococcus aureus also acts as a major human pathogen. The species can cause a broad range of infections, most frequently skin and soft tissue infections, such as wound infections, furuncles, carbuncles and abscesses, but also life-threatening systemic infections, such as pneumonia and sepsis [1-3]. Furunculosis is a common staphylococcal skin disease characterised by painful, deep infections of the hair follicle. Even mild lesions are painful and unsightly and often leave a scar after they heal [4]. Antibiotic treatment is frequently not effective, and many furunculosis patients suffer from recurrent episodes or develop chronic symptoms [4]. 
The alarming global spread of antibiotic resistant strains has spurred efforts to develop active and passive antistaphylococcal vaccines $[5,6]$. However, vaccine development is a challenging task, because both the species $S$. aureus and the host response that it induces are highly variable. Two $S$. aureus strains can differ drastically in their virulence gene content [7]. The variable genome consists of mobile genetic elements such as pathogenicity islands and phages that encode numerous virulence factors, including toxins, exoenzymes and immune modulators [7-10]. In concert with conserved virulence factors, these variable bacterial compounds could determine differential pathogenesis [11]. We observed a strong and strain-specific antibody response against these variable antigens in $S$. aureus carriers and during the natural course of $S$. aureus bacteremia [12].

Active vaccination can be based on mono- or multivalent subunit vaccines or on whole cell vaccines, which include autologous vaccines (short: autovaccines) [6]. Autovaccines are individually prepared from the autologous infecting bacterial strain $[13,14]$. Following subculture, the bacteria and their secreted proteins are usually inactivated by fixation, heat or cell lysis, and then repeatedly applied orally or subcutaneously $[13,15]$. In contrast to subunit vaccines, autovaccines contain poorly characterized variegated cocktails of surface proteins and secreted virulence factors produced by the infecting strain.

Before the antibiotic era, chronic staphylococcal infections such as chronic furunculosis and osteomyelitis were frequently treated by therapeutic vaccination with autologous formalin-killed $S$. aureus cells [14, 16-18]. Today, autovaccination is still regularly performed in some Eastern European countries, including Poland and the Czech Republic $[13,19]$. It is offered as a therapeutic alternative to patients with chronic $S$. aureus infections that are refractory to standard therapy. Moreover, bacterial whole cell vaccines are commonly used in veterinary medicine to treat chronic infectious diseases [20-22]. The major argument against the use of autovaccines in human medicine is that safety and efficacy have not been determined in controlled clinical trials. Moreover, the mode of action is largely unknown.

In this prospective pilot study we analyzed if autovaccination influences the serum antibody response to a broad spectrum of secreted and surface-bound $S$. aureus antigens.

\section{Materials and methods}

Autovaccination patients

The Department of Medical Microbiology and Immunology at the Pomeranian Medical University, Szczecin, Poland has long-standing experience with autovaccination for therapy of chronic S. aureus furunculosis and osteomyelitis [13]. This prospective pilot study included four patients (3 female and 1 male) from the Szczecin area. They suffered from chronic or recurrent furunculosis and asked for autovaccination, one of the treatment options in Poland. In all four patients previous antibiotic treatment and surgical intervention had been unsuccessful. Patient data and anatomic location of the furuncles are shown in Table 1. The patients showed no signs of immune suppression. All four patients provided their written informed consent and the study was approved by the Ethics Board of the University of Szczecin.

\section{S. aureus strains and sera}

The $S$. aureus vaccine strains were isolated from infected lesions (pus) during episodes of furunculosis. Moreover, nasal swabs were taken repeatedly to determine the nasal colonization status. S. aureus was identified on the basis of generally accepted criteria: the presence of clumping factor (slide test) and extracellular coagulase (tube test), and biochemical identification (ID 32 Staph, BioMerieux). The infecting and colonizing $S$. aureus isolates were stored as glycerol stocks.

Antimicrobial susceptibility of isolates was estimated by the disc diffusion method according to current recommendations by the NCCLS/CLSI. Resistance to oxacillin/ cephoxitin was confirmed by detection of PBP $2^{\prime}$ (Slidex MRSA detection, BioMerieux) and mecA PCR (see below).

Sera were obtained directly before and during autovaccination treatment and stored at $-80^{\circ} \mathrm{C}$. We obtained four consecutive serum samples from patient 6466 (day 0,86 , 273 and 332), three samples from patients 7293 (day 0, 42, and 187) and 9105 (day 0,85, and 166), and two samples from patient 7510 (day 0, and 63). Additionally, control sera from 11 healthy lab workers from the Department of Microbiology and Immunology, Pomeranian Medical University, Szczecin (age 37.6 \pm 10.7 years; 36\% male) were analyzed.

Preparation of autologous vaccines

Autovaccines were individually prepared from the causative $S$. aureus strain, which was isolated from furunculosis lesions. Autologous strains were cultivated for a maximum of $24 \mathrm{~h}$ on oblique test tubes containing PPLO agar (Becton Dickinson) supplemented with $10 \%(\mathrm{w} / \mathrm{v})$ glucose and $0.1 \%(\mathrm{v} / \mathrm{v})$ Tween 80 . Bacteria were resuspended in $0.9 \%$ $\mathrm{NaCl}$ solution and fixed with $0.4 \%$ formalin solution for $48 \mathrm{~h}$. The bacterial suspension was adjusted to $5 \times 10^{8}$ bacteria/ml (suspension I), $1 \times 10^{9}$ bacteria/ml (suspension II), and $2.5 \times 10^{9}$ bacteria/ml (suspension III) based on 
Table 1 Clinical outcome of autovaccination

\begin{tabular}{|c|c|c|c|c|c|}
\hline \multicolumn{2}{|l|}{ Patient data } & Patient 6466 & Patient 7293 & Patient 7510 & Patient 9105 \\
\hline \multicolumn{2}{|l|}{ Age (y) } & 23 & 35 & 50 & 41 \\
\hline \multicolumn{2}{|l|}{ Gender } & Female & Female & Male & Female \\
\hline \multicolumn{2}{|l|}{ Risk factors ${ }^{\mathrm{a}}$} & Atopic dermatitis & - & - & Atopic dermatitis \\
\hline \multicolumn{2}{|l|}{ Onset of furuncles } & $02 / 06$ & $07 / 06$ & 2002 & $07 / 06$ \\
\hline \multicolumn{2}{|l|}{ AV treatment } & 08/07-12/08 & $10 / 06-08 / 07$ & $10 / 06-10 / 07$ & $12 / 06-10 / 07$ \\
\hline \multicolumn{2}{|l|}{ Location of furuncles } & Face, neck, axilla & Axilla, abdomen, back & Face, neck & Axilla, buttocks \\
\hline \multirow[t]{3}{*}{ Mean number of episodes per month } & Before AV & 2 & 4 & 2 & 3.5 \\
\hline & During AV & 0 & 1 & 2 & 0.2 \\
\hline & After AV & 1 & 0 & 0.6 & 0 \\
\hline \multirow[t]{3}{*}{ Chronicity } & Before AV & Intermittently & Permanently & Permanently & Permanently \\
\hline & During AV & Symptom free & Intermittently & Intermittently & Intermittently \\
\hline & After AV & Intermittently & Symptom free & Intermittently & Symptom free \\
\hline \multirow[t]{3}{*}{ Single/multiple sites } & Before AV & Multiple & Multiple & Multiple & Multiple \\
\hline & During AV & - & Multiple(1 episode) & Single & Single \\
\hline & After AV & Single & - & Single & - \\
\hline \multirow[t]{3}{*}{ Severity score ${ }^{b}$} & Before AV & 2 & 2 & 2 & 3 \\
\hline & During AV & 0 & 1 & 1 & 1 \\
\hline & After AV & 1 & 0 & 1 & 0 \\
\hline \multicolumn{2}{|l|}{ Clinical improvement $^{\mathrm{c}}$} & Strong & Strong & Moderate & Strong \\
\hline
\end{tabular}

$A V$ autovaccination

${ }^{a}$ Risk factors: atopic dermatitis, chronic wounds, gout, diabetes mellitus, COPD, cancer, chronic liver disease, cardiovascular disease, rheumatoid diseases, HIV, chronic gut diseases (Morbus Crohn, Ulcerative Colitis), psoriasis, regular dialysis, immune suppressive drugs

${ }^{\mathrm{b}}$ Disease severity was assessed based on a severity score. 0 - No furuncles/abscesses; 1 - Few small furuncles, self-healing in less than two weeks, no medical intervention required; 2 - Furuncles of moderate severity, medical intervention required; 3 - Severe furuncles/carbuncles with strong pain and large fluid collections, medical intervention required

${ }^{\mathrm{c}}$ Clinical improvement (classified as none, moderate or strong) based on the patients personal rating.

McFarland quantification. Afterwards, $0.1 \mathrm{mg} / \mathrm{ml}$ thiomersal was added to these suspensions as preservative. Finally, sterility of autovaccine preparation was tested by cultivation in thioglycollate medium (Becton Dickinson) for 7 days.

\section{Administration of autovaccines}

Autovaccines were prepared by the Department of Microbiology and Immunology, Pomeranian Medical University in Szczecin, and administered by the patients' general practitioners. Before the beginning of treatment, a skin test was performed to exclude hypersensitivity of the patient to any of the vaccine components. Intradermally, $0.5 \mathrm{ml}$ of a 1:10 dilution of suspension I in Aqua ad injectionem was injected. Skin reaction was checked after $15 \mathrm{~min}$ and 24 hours. Autovaccines were then applied subcutaneously in 3-5 day intervals over 3 months. With each injection the dose was increased based on the following application scheme. Suspension I $\left(5 \times 10^{8}\right.$ bacteria $\left./ \mathrm{ml}\right)$ was applied in volumes of $0.1,0.2,0.3,0.4$ and $0.5 \mathrm{ml}$, followed by suspension II $\left(1 \times 10^{9}\right.$ bacteria $/ \mathrm{ml} ; 0.3,0.4,0.5,0.6,0.7$, $0.8,0.9,1.0 \mathrm{ml})$ and suspension III $\left(2.5 \times 10^{9}\right.$ bacteria $/ \mathrm{ml}$; $0.5,0.6,0.7,0.8,0.9,1.0 \mathrm{ml})$. This immunization procedure was followed by several recall injections (suspension III, $1 \mathrm{ml}$ ) every 1-3 weeks for up to 5-12 months. After each injection the patients were closely monitored for 30 minutes (swelling, pain or rash on injection side, blood pressure, breathing). After every vaccination, presumptive systemic symptoms (fever, headache, general malaise, sore throat, etc.) were also analyzed by a physician to exclude any side effects associated with autovaccination. Moreover, laboratory tests were performed before autovaccination and after completion of the basic application scheme (blood count, $\mathrm{CRP}$; urine: proteinuria).

\section{Clinical evaluation}

The impact of autovaccination on the severity and frequency of furunculosis was retrospectively analyzed by reviewing the clinical records. Additionally, a telephone interview using a questionnaire was performed by 
a physician, which included questions about the chronicity, duration, location and severity of furuncles, as well as a patient's rating of the clinical improvement after autovaccination.

Detection of $S$. aureus virulence factors by PCR

PCR was used to screen for a total of 26 virulence genes. Single and multiplex-PCR were applied for the detection of gyrase (gyr), methicillin resistance (mecA), PantonValentine-leukocidin $(p v l)$, staphylococcal enterotoxins (sea-seu), toxic shock syndrome toxin 1 (tst), exfoliative toxins (eta, etb, etd), and agr groups 1-4 as previously reported [8].

\section{spa genotyping}

PCR for amplification of the S. aureus protein A (spa) repeat region was performed according to the published protocol [23, 24]. PCR products were sequenced by a commercial supplier using both amplification primers (Agowa, Berlin, Germany). The forward and reverse sequence chromatograms were analyzed with the Ridom StaphType software (Ridom GmbH, Würzburg, Germany). Spa types were clustered into different clonal clusters using the Based Upon Repeat Pattern (BURP) algorithm. Since spa typing and multilocus-sequence typing (MLST) are highly concordant, spa typing data could be mapped on MLST types by using the SpaServer database (www. spaserver.ridom.de).

\section{D-Immunoblots}

Two-dimensional polyacrylamide gel electrophoresis (2-DE) with mini 2D gels and 2D immunoblots (2D-IBs) were performed as described [25]. Briefly, for antigen preparation, bacteria were inoculated in tryptic soy broth (TSB) to an optical density at $540 \mathrm{~nm}$ of 0.05 and cultivated in $100 \mathrm{ml}$ cultures at $37^{\circ} \mathrm{C}$ and $180 \mathrm{rpm}$. Cultures were harvested $3.5 \mathrm{~h}$ after the bacterial culture entered the stationary phase, extracellular proteins were collected, and protein concentration was determined as previously described [11, 25].

Afterwards, isoelectric focusing was performed with $7 \mathrm{~cm}$ Immobiline Dry Strips of pI ranges 4-7 and 6-11 (GE Healthcare, Munich, Germany). Following separation according to molecular mass in the second dimension, the resolved staphylococcal proteins were blotted onto a PVDF membrane (Immobilon-P, Millipore) and incubated with the corresponding human sera at 1:10,000 dilution. IgG binding was detected by peroxidase-conjugated goat anti-human IgG and visualized with an ECL substrate (SuperSignal West Femto Maximum Sensitivity Substrate, Pierce). Consecutive serum samples from one patient were always analyzed simultaneously; at least three technical replicates were performed for each patient.

Analysis of the 2D-IB images was performed with the Delta-2D software package version 4.0 (Decodon $\mathrm{GmbH}$, Greifswald, Germany) as described [11, 25]. A fused image of all IBs from one patient was obtained in a two-step procedure. First, all IB images from one time course experiment were matched with the most complex IB and a fusion image was obtained using the union fuse option. Second, the fusion images from the three technical replicates were matched and fused. Spots on the fusion image were automatically detected and manually validated by comparing the original blot images with the fusion image. Subsequently, the spot map and the corresponding labels from the fusion image were transferred to all blot images from one patient. For spot detection on IBs the signal intensity threshold was set to 0.2 arbitrary units (AU).

Flamingo ${ }^{\circledR}$ protein staining

To correlate IB binding with protein abundance, representative IB images were matched with Flamingo ${ }^{\circledR}$-stained 2D gels. Gels were stained with the Flamingo ${ }^{\circledR}$ Fluorescent Gel Stain (BioRad, Munich, Germany) according to the manufacturer's instructions.

Bead-based flow cytometry (Luminex ${ }^{\circledR}$ )

Serum IgG, IgA and IgM directed against a panel of 40 recombinant staphylococcal antigens was quantified using the bead based flow cytometry technique (xMap ${ }^{\circledR}$, Luminex Corporation, Austin, Texas, USA). Antigens comprised surface proteins, such as clumping factor A and B (ClfA, $\mathrm{ClfB}$ ), and serine-aspartate dipeptide repeat proteins ( $\mathrm{SdrD}$ and SdrE), and secreted proteins, including toxins (superantigens, leukotoxins) and immune modulatory proteins (CHIPS, SCIN, SSL proteins).

Methods were as described previously [26, 27]. Tests were performed in independent duplicates and the median fluorescence intensity (MFI) values, reflecting antibody concentrations, were averaged. In each experiment, control beads (no protein coupled) were included to determine nonspecific antibody binding. In case of non-specific binding, this background was subtracted from the MFI values. Human pooled serum was used as a standard.

\section{Statistics}

Differences in antibody binding (Luminex ${ }^{\circledR}$ ) between furunculosis patients and healthy controls were assessed using the Mann-Whitney $U$ test in conjunction with the Benjamini-Hochberg false discovery rate (FDR) multiple testing correction $(\mathrm{FDR}<0.05)$. 


\section{Results}

Clinical outcome of autovaccination

To assess the impact of autovaccination on the severity and frequency of furunculosis, clinical records were screened and telephone interviews using a questionnaire were performed by a physician (Table 1). All four patients suffered from chronic (7293, 7510, and 9105) or recurrent (6466) furunculosis, which required repeated medical interventions.

Autovaccination reduced the frequency and severity of furunculosis or resulted in complete remission (Table 1). Two patients (7293 and 9105) were free of symptoms after autovaccination treatment. The other two $(6466,7510)$ still developed single, self-healing furuncles, which did not require medical treatment. All patients reported subjective improvement (Table 1). Side effects occasionally observed by the patients were pain and inflammation at the side of injection and elevated temperature $\left(<38^{\circ} \mathrm{C}\right)$ for two days after injection.

\section{Molecular characterization of the furunculosis strains}

The infecting $S$. aureus strains were repeatedly isolated from infected skin lesions. In parallel, nasal swabs were taken to determine the nasal colonization status. The infecting and colonizing $S$. aureus isolates were characterized by spa genotyping. Additionally, a panel of virulence genes, including $p v l$, mecA, exfoliative toxins and superantigens was detected by multiplex PCR (Table 2).
Three patients were nasal $S$. aureus carriers. Molecular characterization showed that all $S$. aureus furunculosis and nasal strains isolated from one patient over time belonged to the same $S$. aureus clone. Notably, two patients (7293 and 9105) were infected with a community-acquired methicillin-resistant $S$. aureus strain (CA-MRSA), which belonged to the clonal cluster CC59. Both strains harbored the characteristic mecA and $p v l$ genes and displayed a macrolide-lincosamide-streptogramin B resistance $\left(\mathrm{MLS}_{\mathrm{B}}\right)$ phenotype. Nasal and infecting strains from patient 6466 were $p v l$-positive and belonged to $\mathrm{CC} 121$, a lineage strongly associated with furunculosis [28]. Patient 7510 carried a $p v l$-negative CC8 isolate (Table 2).

Anti-staphylococcal serum antibodies before autovaccination

Taking into account the pronounced variability of the species $S$. aureus we used a personalized approach to study the kinetics of the human antibody response in autovaccination. Two-dimensional immunoblots (2D-IB) served to analyze the binding of each patient's serum antibodies to the antigen spectra of the corresponding infecting $S$. aureus strain. Our analyses were focused on the more informative $\mathrm{pH}$ range of $6-11$, where most extracellular proteins resolve, while protein $\mathrm{A}$ is excluded, so that unspecific IgG binding could be avoided [25].

The furunculosis patients showed a strong antibody response against the extracellular proteins of their infecting $S$. aureus strain already before autovaccination treatment (Fig. 1a). All four patients showed a relatively complex

Table 2 Virulence genes and genotype of $S$. aureus strains

\begin{tabular}{|c|c|c|c|c|c|c|c|c|c|c|c|}
\hline \multicolumn{4}{|c|}{ Clinical data } & \multicolumn{6}{|c|}{ Virulence genes ${ }^{\mathrm{a}}$} & \multicolumn{2}{|l|}{ Genotype } \\
\hline Patient & S. aureus isolate & Date & Source & mecA & $p v l$ & non-egc SAgs & $e g c \mathrm{SAgs}$ & eta, etd & $a g r$ & spa type & Deduced MLST CC \\
\hline 6466 & $6466 / 1$ & 22.06 .07 & $\mathrm{Pus}^{\mathrm{c}}$ & - & + & - & gimnou & - & 4 & t645 & $\mathrm{CC} 121$ \\
\hline 6466 & $6466 / 2$ & 20.08 .07 & Nose & - & + & - & gimnou & - & 4 & t645 & $\mathrm{CC} 121$ \\
\hline 6466 & $6466 / 2 a$ & 14.11 .07 & Nose & - & + & - & gimnou & - & 4 & t645 & $\mathrm{CC} 121$ \\
\hline 6466 & $6466 / 2 b$ & 17.07.08 & Nose & - & + & - & gimnou & - & 4 & t645 & $\mathrm{CC} 121$ \\
\hline 7293 & $7293 / 1$ & 20.09 .06 & $\mathrm{Pus}^{\mathrm{c}}$ & + & + & - & - & - & 1 & $\mathrm{t} 437$ & CC59 \\
\hline 7293 & $7293 / 2$ & 20.09 .06 & Nose & + & + & - & - & - & 1 & $\mathrm{t} 437$ & CC59 \\
\hline 7293 & $7293 / 2 \mathrm{a}$ & 12.12 .06 & Nose & + & + & - & - & - & 1 & $\mathrm{t} 437$ & CC59 \\
\hline 7510 & $7510 / 1$ & 27.09 .06 & $\mathrm{Pus}^{\mathrm{c}}$ & - & - & - & gimnou & - & 4 & $\mathrm{t} 037$ & $\mathrm{CC} 8$ \\
\hline 7510 & $7510 / 2$ & 27.09 .06 & Nose & - & - & - & gimnou & - & 4 & t037 & $\mathrm{CC} 8$ \\
\hline 7510 & $7510 / 1 b$ & 20.12 .06 & Pus & - & - & - & gimnou & - & 4 & $\mathrm{t} 037$ & $\mathrm{CC} 8$ \\
\hline 9105 & $9105 / 1$ & 20.11 .06 & $\mathrm{Pus}^{\mathrm{c}}$ & + & + & $b k q$ & - & - & 1 & $\mathrm{t} 437$ & CC59 \\
\hline
\end{tabular}

${ }^{a}$ Staphylococcal enterotoxins (SEs) are indicated by single letters $(a=s e a$, etc.). $t s t=$ toxic shock syndrome toxin, $e g c=$ enterotoxin gene cluster, eta, etd $=$ exfoliative toxins a and $\mathrm{d}, a g r=$ accessory gene regulator, $p v l=$ Panton-Valentine leucocidine $(l u k P V)$.

${ }^{\mathrm{b}}$ MLST CC nomenclature was deduced from spa types using the Ridom SpaServer database.

${ }^{\mathrm{c}}$ The strain isolated from purulent furuncles was used to prepare the autovaccine 
antibody binding pattern, especially in the basic $\mathrm{pH}$ range. However, there were also numerous protein spots which were not recognized by serum IgG. IB images from $\mathrm{pH}$ 4-7 were less complex and a number of spots were due to $\mathrm{IgG}$ binding to protein $\mathrm{A}$. Overall, they corroborate the observations made in $\mathrm{pH}$ range 6-11 (data not shown).
Next, Luminex ${ }^{\circledR}$ technology was used to compare the antibody response in furunculosis patients (before autovaccination) and healthy controls. Anti-staphylococcal $\mathrm{IgG}$, $\operatorname{IgA}$ and $\operatorname{IgM}$ serum concentrations in the four investigated patients were comparable to those in healthy individuals $(n=11)$ (Fig. 2). Serum $\operatorname{IgG}$ and $\operatorname{IgA}$ from furunculosis patients reacted with a broad range of staphylococcal
A
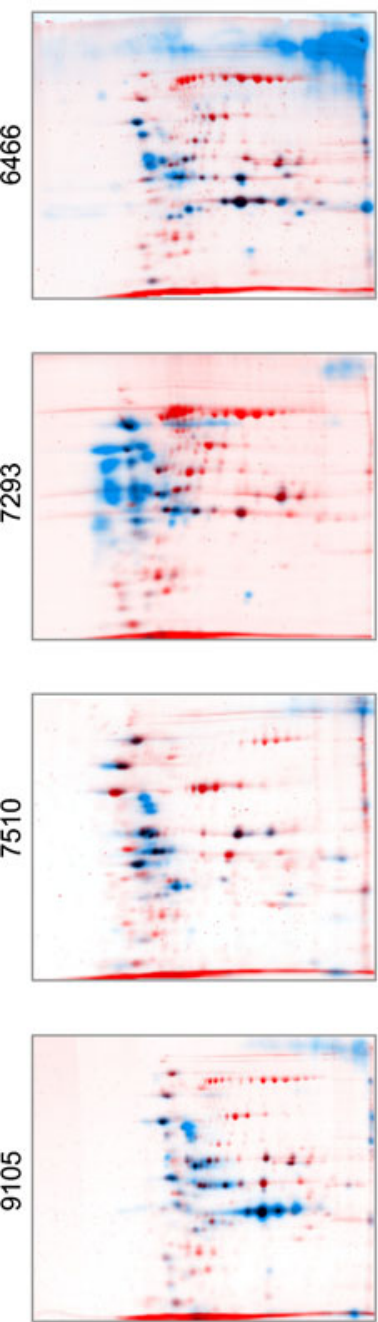

Serum 1

Protein
B
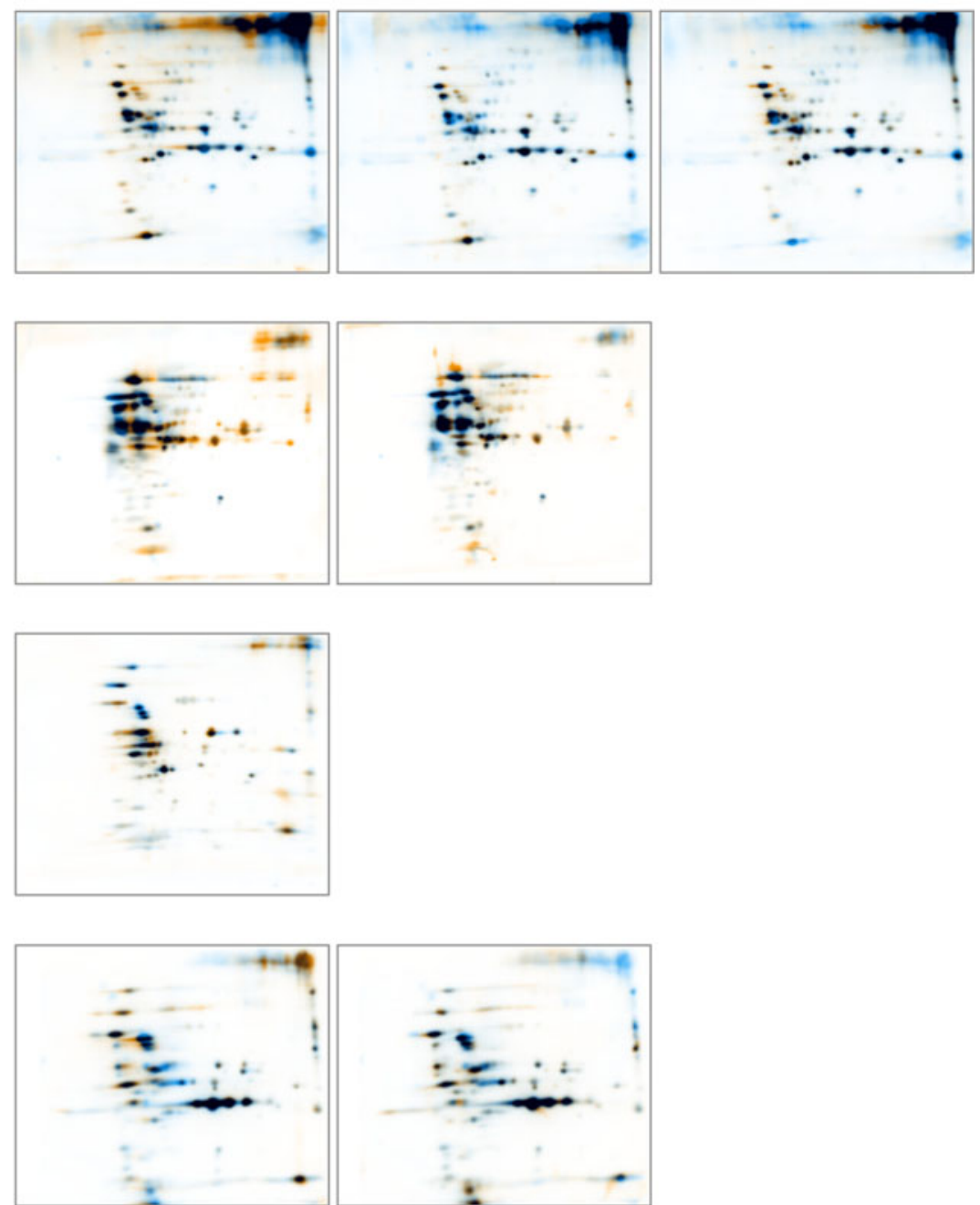

Serum 1

Serum 2

Serum 1

Serum 3

Serum 1

Serum 4

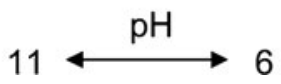

Fig. 1 Serum IgG response to extracellular $S$. aureus proteins in autovaccination. a Overlay images of Flamingo ${ }^{\circledR}$-stained 2D gels and corresponding 2D-immunoblots. The proteins released during postexponential growth by the four invasive $S$. aureus strains were separated by $2 \mathrm{D}$ gel electrophoresis $(\mathrm{pH} 6-11)$ and stained with the fluorescence protein dye Flamingo ${ }^{\circledR}$ ( $\left.r e d\right)$. The four bacterial isolates differed in their secretomes. In parallel, the extracellular bacterial antigens were blotted onto PVDF membranes and incubated with the corresponding patients' sera obtained before autovaccination. The overlay images (protein - red; IgG binding - blue) showed a strong antibody response to the infecting strain already before autovaccination. b Kinetics of individual $\mathrm{IgG}$ responses to autovaccination. Extracellular antigens from the infecting $S$. aureus strains were separated by 2D gel electrophoresis and quantitative IBs were performed with the corresponding patients' sera obtained at different time points. Overlays of 2D-IBs with sera obtained before (blue) and during (orange) autovaccination. Autovaccination induced a moderate boost in the $\mathrm{IgG}$ response. One out of the three technical replicates is shown 
surface antigens, toxins (e.g. PVL, superantigens) and immunomodulatory proteins. There was little IgM binding to staphylococcal antigens in controls as well as two patients, but strong binding to many $S$. aureus antigens in the other two patients (Fig. 2). Superantigens encoded by the enterotoxin gene cluster, $e g c$, were on average bound less strongly than others. To conclude, furunculosis patients did not have a general lack of anti-staphylococcal antibodies.

Antibody response during autovaccination

To test whether autovaccination triggers an antibody response, we studied the kinetics of antibody binding using 2D-IBs and Luminex. First, we performed 2D-IBs with sera obtained over the course of autovaccination (2-4 samples). Superimposition of 2D-IB images from serum samples obtained before autovaccination (blue) and later (orange) showed almost identical antibody binding patterns as indicated by the dominance of black spots on the superimposed images (Fig. 1b). Only a few new IgG-binding specificities, indicated by purely orange spots, were generated. Patient 7293 was exceptional, because we observed a boost in the IgG-binding as well as numerous novel IB-spots.

Next, we quantified the individual IB-spot intensities and determined the changes in antibody binding during autovaccination (Fig. 3). The patients' sera obtained before autovaccination reacted with $55-82$ protein spots. Patient 7293 showed the weakest antibody binding before vacci-
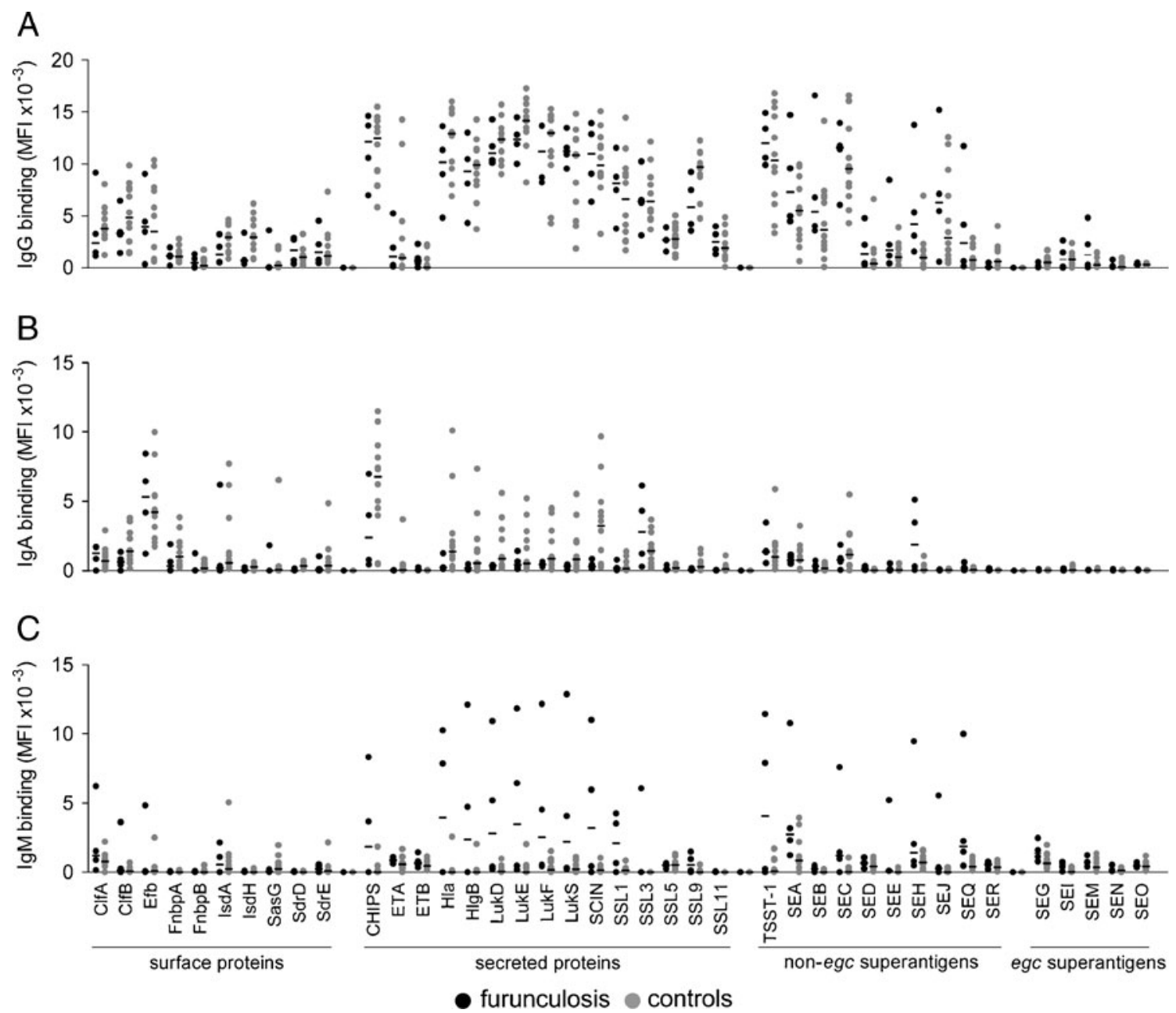

Fig. 2 Serum $\operatorname{IgG}, \operatorname{IgA}$ and $\operatorname{IgM}$ response to $S$. aureus surface proteins and toxins in furunculosis patients and healthy controls. Serum $\operatorname{IgG}(\mathbf{a}), \operatorname{IgA}$ (b) and $\operatorname{IgM}$ (c) antibody binding to a panel of 40 S. aureus antigens was assessed with a Luminex assay and Ig binding to individual antigens is depicted as median fluorescence intensity (MFI). Each symbol represents a single individual. Median levels of anti-staphylococcal antibodies for patients ( $n=4$; before autovaccination) and healthy controls $(n=11)$ are indicated. The antibody binding patterns of furunculosis patients and healthy controls were not significantly different (Mann-Whitney $U$ test using the BenjaminiHochberg false discovery rate multiple testing correction). Abbreviations: ClfA Clumping factor A, Efb extracellular fibrinogen-binding protein, FnbpA Fibronectin binding protein A, IsdA iron-responsive surface determinant, Sas $S$. aureus surface protein, $S d r$ serine-aspartate dipeptide repeat proteins, CHIPS chemotaxis inhibitory protein of $S$. aureus, Luk leukocidin, SCIN staphylococcal complement inhibitor, $S S L$ staphylococcal superantigen-like protein, $S E$ staphylococcal enterotoxin 
nation (Fig. 3a). In this patient, vaccination induced a two- to five-fold intensity increase in most spots (59/67) (Fig. 3b). A moderate boost was observed in patient 7510 with $22 / 83$ spots increasing more than two-fold. The antibody patterns of the other two patients (6466 and 9105) remained almost unchanged (Fig. 3b). Notably, autovaccination induced only few novel antibody specificities. In conclusion, autovaccination boosted the IgG response to extracellular antigens in two patients, while the antibody response of the other two patients was not affected.

Finally, we applied the Luminex assay to test whether autovaccines trigger an antibody response to surface-bound staphylococcal antigens. Indeed, three out of four patients showed a more than two-fold increase in IgG binding to surface antigens, such as ClfA (7293, 7510, 9105), ClfB (9105), SdrD (7510) and SdrE (7293) (Fig. 4). Similarly, we observed a boost in the IgA response against surface proteins (suppl. Fig. 1). Thus, vaccination induced a moderate boost of the antibody response against staphylococcal surface proteins. Novel antibody specificities against surface proteins were not generated.

\section{Discussion}

Autovaccines have been applied in chronic staphylococcal infections for decades, but their mechanism of action is still unknown. In this pilot study, we demonstrate that autovaccination only slightly boosts the antibody response to extracellular bacterial antigens and surface proteins.

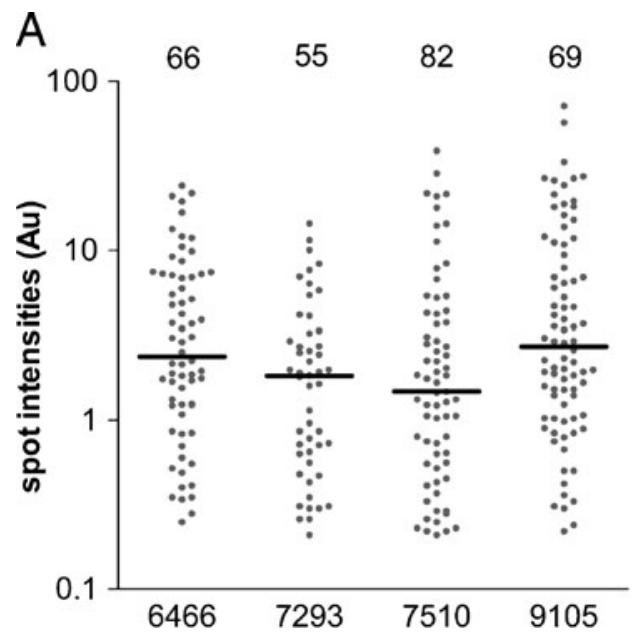

Fig. 3 Quantification of $\operatorname{IgG}$ binding to extracellular $S$. aureus antigens. Spot intensities on $2 \mathrm{D}-\mathrm{IB}$ were determined from three or more replicate blots and median intensities in arbitrary units (AU) are depicted. The threshold was set at a volume of 0.2 AU. a Spot intensities before autovaccination. Before autovaccination, we detected strong serum IgG binding to numerous antigens from the infecting strain. The total number of immunoblot spots is displayed above each data set. Median intensities from all spots are indicated. b
This pilot study was not designed to test the efficacy of autovaccination, but rather to characterize the kinetics of the antibody responses. Therefore, we analyzed only a small cohort and did not include placebo controls. Moreover, some clinical outcome criteria (severity score, rating of clinical improvement) are subjective, which limits the evaluation of the clinical outcome. Nevertheless, it is interesting to note that based on the applied criteria all four patients, two of whom were chronically infected with CA-MRSA, clinically improved during treatment. In the 1970s, Ring et al. reported that oral autovaccination treatment improved the clinical state in $11 / 18$ patients with chronic posttraumatic osteomyelitis [15]. Similarly, a controlled study of 292 children with paranasal sinusitis demonstrated that antibiotic therapy plus autovaccine was superior to antibiotic therapy alone [29]. Overall, little is published in English on the efficacy and safety of autovaccination. In particular, prospective, controlled, randomized, and, if possible, (double)-blind clinical studies are lacking.

Already before autovaccination, the study patients had serum antibodies that bound to a broad antigen spectrum of their infecting $S$. aureus strain. There was no obvious defect in their anti-staphylococcal antibody response. Furunculosis patients suffer from chronic or recurrent infection for many months or even years and, therefore, the immune system is frequently and intensively exposed to the invasive strain and establishes a memory response. Moreover, most furunculosis patients are $S$. aureus carriers and the nasal and infecting strains are usually identical

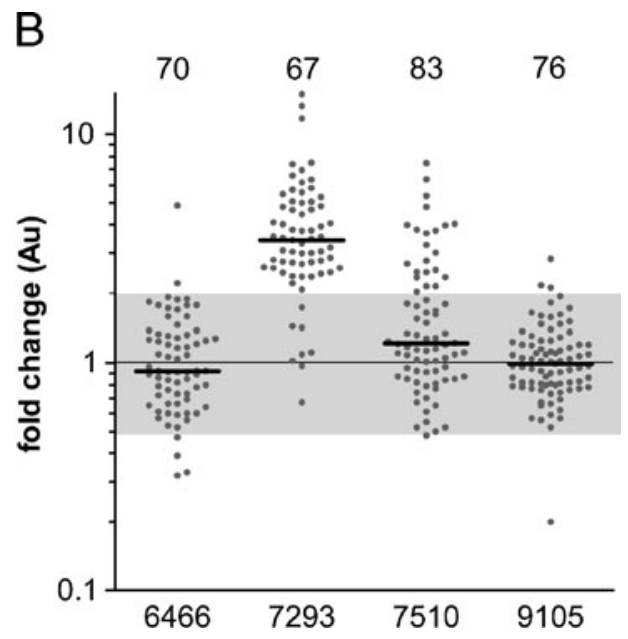

Changes of spot intensities during autovaccination. To monitor changes in IgG binding, the ratios between the last and first serum sample were calculated for each spot (ratios of median spot intensities). In patient 7293 most spots showed a two- to five-fold increase in $\operatorname{IgG}$ binding. Shaded area corresponds to a two-fold change. The total number of immunoblot spots obtained with the last serum is displayed above each data set. The overall medians are depicted 

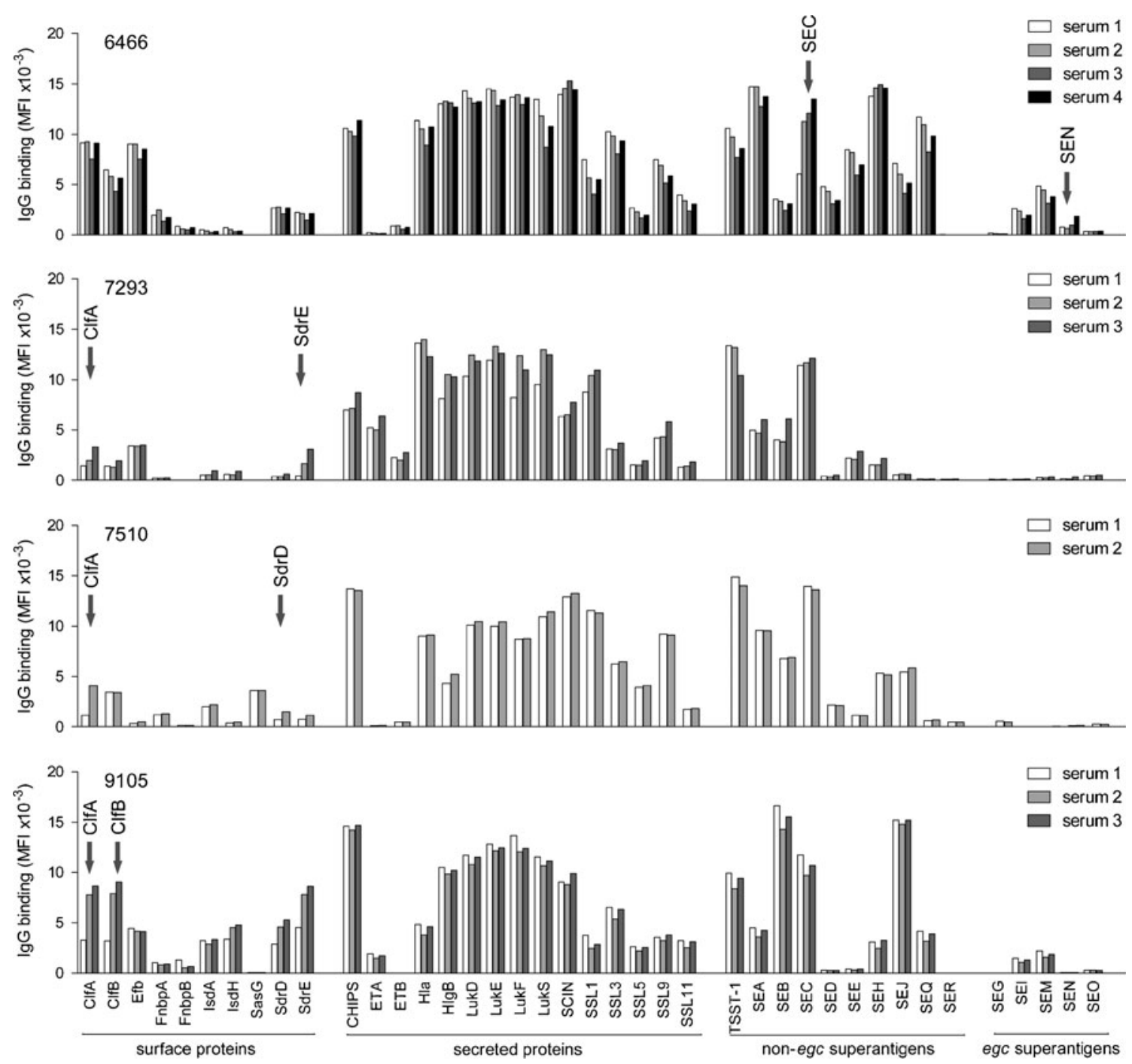

Fig. 4 Kinetics of the serum $\operatorname{IgG}$ response to $S$. aureus surface proteins and toxins over the course of autovaccination. IgG binding to a panel of $40 \mathrm{~S}$. aureus antigens was assessed with a Luminex assay. Each graph represents a single patient. Per patient, the level of antigen-specific antibodies in the time course of autovaccination is shown. Arrows indicate a more than two-fold increase in antibody binding. See legend to Fig. 2 for more detailed information on the antigens
[28]. In this study, three out of four patients were colonized with the infecting strain in the nose. Thus, the nose appears to be a potential source for repeated endogenous infections. In agreement with our findings of a robust baseline anti-S. aureus antibody response in the furunculosis patients, we previously reported that healthy human carriers are pre-immunized with their colonizing strain $[12,25]$.

By applying 2D-IB and Luminex assays we found that autovaccination only slightly boosted the pre-existing antibody response to secreted bacterial antigens and surface proteins. Notably, autovaccination did not induce a profound de novo generation of antibody specificities. These findings suggest that repeated subcutaneous immunization does not strongly trigger the humoral immune response in these well pre-immunized patients. In line with this, Szkaradkiewicz et al. analyzed the IgG response in patients treated with autovaccines using 1D-immunoblots and also observed no changes in IgG binding patterns to $S$. aureus polypeptides [30]. In contrast to autovaccination of furunculosis patients, systemic $S$. aureus infection strongly enhances the anti-staphylococcal antibody response in patients [10].

We previously reported a high degree of strainspecificity in the anti-S. aureus antibody response [12]. However, it is also possible that some antibody specificities are cross-reactive. For example, patient 6466 mounted an antibody response against SEC, but the strain used for 
autovaccination lacked the sec gene. SEC belongs to a family of 21 staphylococcal superantigens with up to $90 \%$ sequence homology, which could explain the observed cross-reactivity [31, 32].

The pore-forming toxin PVL is epidemiologically strongly linked to $S$. aureus furunculosis $[3,28]$. In this study, three out of four patients were infected with $p v l$ positive strains. All patients had high levels of $\operatorname{IgG}$ antibodies against both subunits of the toxin, LukF and LukS, which did not increase during autovaccination.

Little is known about the mechanisms by which autovaccines might influence the disease course or impact on the immune response. Halasa et al. reported that autovaccination increases the concentration of $S$. aureus strain-specific agglutinating antibodies and enhances the phagocytosis of bacteria by peripheral blood granulocytes [13]. Similarly, clinical improvement of acne after autovaccination with killed Propionibacterium acnes was accompanied by the generation of specific antibodies against bacterial structures [33]. Still, it is difficult to imagine that the observed moderate changes of antibody titers should fully explain the clinical effects of the treatment. Other groups have reported that autovaccination down-regulates Th1 cell function and reduces delayed type hypersensitivity reactions [15, 21, 34]. In conjunction with the observation that $\mathrm{T}$ cells can influence the recruitment and anti-bacterial action of granulocytes [35, $36]$, this opens an interesting perspective for research. Moreover, the humoral immune response to non-protein staphylococcal antigens, such as lipoteichoic acid, wall teichoic acid and capsular polysaccharides, might be clinically important and will be addressed in future analyses [11, 37]. Finally, we cannot rule out that formalin-fixation of the vaccine preparation influences the three-dimensional antigen structure, which might influence the antibody response, especially to surface antigens.

The rapid spread of multiresistant $S$. aureus strains enforces the development of new strategies to combat staphylococcal infections. In view of the pronounced variability of the bacterial species, personalized approaches could hold promise for patients with recurrent or chronic $S$. aureus infection. Autovaccination could serve as a model that merits further investigation.

Acknowledgements This work was supported by the Deutsche Forschungsgemeinschaft (SFB-TR34 "Staphylococci in the postgenomic era", GRK840 "Host-Pathogen Interactions in Generalized Bacterial Infections").

Open Access This article is distributed under the terms of the Creative Commons Attribution Noncommercial License which permits any noncommercial use, distribution, and reproduction in any medium, provided the original author(s) and source are credited.

\section{References}

1. Dryden M (2008) Complicated skin and soft tissue infections caused by methicillin-resistant staphylococcus aureus: epidemiology, risk factors, and presentation. Surg Infect (Larchmt) 9(Supp 1):s3-s10

2. Lina G, Piemont $\mathrm{Y}$, Godail-Gamot F, Bes M, Peter M, Gauduchon V, Vandenesch F, Etienne J (1999) Involvement of Panton-Valentine leukocidin-producing Staphylococcus aureus in primary skin infections and pneumonia. Clin Infect Dis 29(5):11281132

3. Wertheim HF, Vos MC, Ott A, van Belkum A, Voss A, Kluytmans JA, van Keulen PH, Vandenbroucke-Grauls CM, Meester MH, Verbrugh HA (2004) Risk and outcome of nosocomial Staphylococcus aureus bacteraemia in nasal carriers versus non-carriers. Lancet 364(9435):703-705

4. Hay RJ, Adriaans B (2004) Bacterial infections. In: Burn T, Breathnach S, Cox N, Griffiths CE (eds) Rook's textbook of dermatology. Blackwell Publishing Company, London, United Kingdom, pp 1-85

5. Boucher HW, Corey GR (2008) Epidemiology of methicillinresistant Staphylococcus aureus. Clin Infect Dis 46(s5):S344-S349

6. Schaffer AC, Lee JC (2009) Staphylococcal vaccines and immunotherapies. Infect Dis Clin North Am 23(1):153-171

7. Lindsay JA, Holden MT (2004) Staphylococcus aureus: superbug, super genome? Trends Microbiol 12(8):378-385

8. Holtfreter S, Grumann D, Schmudde M, Nguyen HT, Eichler P, Strommenger B, Kopron K, Kolata J, Giedrys-Kalemba S, Steinmetz I, Witte W, Broker BM (2007) Clonal distribution of superantigen genes in clinical Staphylococcus aureus isolates. J Clin Microbiol 45(8):2669-2680

9. Novick RP, Subedi A (2007) The SaPIs: mobile pathogenicity islands of staphylococcus. Chem Immunol Allergy 93:42-57

10. Goerke C, Pantucek R, Holtfreter S, Schulte B, Zink M, Grumann D, Broker BM, Doskar J, Wolz C (2009) Diversity of prophages in dominant Staphylococcus aureus clonal lineages. J Bacteriol 191(11):3462-3468

11. Holtfreter S, Kolata J, Broker BM (2010) Towards the immune proteome of Staphylococcus aureus - the anti-S. aureus antibody response. Int J Med Microbiol 300(2-3):176-192

12. Holtfreter S, Roschack K, Eichler P, Eske K, Holtfreter B, Kohler C, Engelmann S, Hecker M, Greinacher A, Broker BM (2006) Staphylococcus aureus carriers neutralize superantigens by antibodies specific for their colonizing strain: a potential explanation for their improved prognosis in severe sepsis. J Infect Dis 193 (9): $1275-1278$

13. Halasa J, Giedrys-Galant S, Podkowinska I, Braun J, Strzelecka G, Dabrowski W (1978) Evaluation of certain immunological parameters in the course of autovaccine treatment in patients with chronic ostitis and carbunculosis. Arch Immunol Ther Exp (Warsz) 26(1-6):589-593

14. Wright AE (1902) Notes on the treatment of furnculosis, sycosis, and acne by the inoculation of a staphylococcus vaccine and generally on the treatment of localised bacterial invasions by therapeutic inoculations of the corresponding bacterial vaccines. Lancet 159(4100):874-884

15. Ring J, van Thiel D, Seifert J, Stickl H, Probst J, Brendel W (1976) Chronic posttraumatic osteomyelitis. Attempt at oral autovaccine therapy. Fortschr Med 94(5):264-265

16. Scott T, Scott G (1912) A record of the treatment of bacterial infections by autogenous vaccines. Lancet 879

17. Goodale RL, Mangiaracine A (1952) Clinical observations on use of autogenous vaccine made from cultures of coagulase positive hemolytic Staphylococcus aureus. Laryngoscope 62:299-311 
18. Lewin J (1935) Therapy of disseminated furunculosis with autogenous and heterogenous vaccines. T Dermat Ztschr 71:85-88

19. Novotny Z, Krupicka J, Hanova I, Rys E (1990) Treatment with autovaccines and the immunologic profile of patients (in Czech). Cesk Otolaryngol 39(1):40-47

20. Cu HP, Nguyen NN, Do NT, Nguyen XH, Au XT, Van TH, Vu NQ, Dao TH (2006) A study of edema disease in pigs in Vietnam with particular reference to the use of autovaccine for the prevention of disease. Ann NY Acad Sci 1081:531-533

21. Nolte O, Morscher J, Weiss HE, Sonntag H (2001) Autovaccination of dairy cows to treat post partum metritis caused by Actinomyces pyogenes. Vaccine 19(23-24):3146-3153

22. Nolte O, Weiss H-E, Sonntag H-G, Hartmann S (20004) Homologe Autovakzination in der Behandlung von Infektionskrankheiten der Pferde - Ergebnisse einer retrospektiven Erhebung. Tierärztl Umsch 59:315-320

23. Harmsen D, Claus H, Witte W, Rothganger J, Turnwald D, Vogel U (2003) Typing of methicillin-resistant Staphylococcus aureus in a university hospital setting by using novel software for spa repeat determination and database management. J Clin Microbiol 41 (12):5442-5448

24. Aires-de-Sousa M, Boye K, de Lencastre H, Deplano A, Enright MC, Etienne J, Friedrich A, Harmsen D, Holmes A, Huijsdens XW, Kearns AM, Mellmann A, Meugnier H, Rasheed JK, Spalburg E, Strommenger B, Struelens MJ, Tenover FC, Thomas J, Vogel U, Westh H, Xu J, Witte W (2006) High interlaboratory reproducibility of DNA sequence-based typing of bacteria in a multicenter study. J Clin Microbiol 44(2):619-621

25. Holtfreter S, Nguyen TT, Wertheim H, Steil L, Kusch H, Truong QP, Engelmann S, Hecker M, Volker U, van Belkum A, Broker BM (2009) Human immune proteome in experimental colonization with Staphylococcus aureus. Clin Vaccine Immunol 16 (11):1607-1614

26. Verkaik N, Brouwer E, Hooijkaas $H$, van Belkum A, van Wamel W (2008) Comparison of carboxylated and Penta-His microspheres for semi-quantitative measurement of antibody responses to His-tagged proteins. J Immunol Methods 335(12):121-125
27. Verkaik NJ, de Vogel CP, Boelens HA, Grumann D, Hoogenboezem T, Vink C, Hooijkaas H, Foster TJ, Verbrugh HA, van Belkum A, van Wamel WJ (2009) Anti-staphylococcal humoral immune response in persistent nasal carriers and noncarriers of Staphylococcus aureus. J Infect Dis 199(5):625-632

28. Masiuk H, Kopron K, Grumann D, Goerke C, Kolata J, Jursa-Kulesza J, Giedrys-Kalemba S, Broker BM, Holtfreter S (2010) Recurrent furunculosis - associations with Panton-Valentine leukocidin and the genetic background of Staphylococcus aureus. J Clin Microbiol

29. Okrasinska-Cholewa B (1994) Clinical evaluation of treating accessory nasal sinus diseases in children using autovaccine. Med Dosw Mikrobiol 46(1-2 Suppl):67-73

30. Szkaradkiewicz A, Kuch A, Korzeniowska-Kowal A, Kuzma A, Gamian A (2005) Humoral response to Staphylococcus aureus polypeptides in patients treated with autovaccine. XXIst ISTH Congress, Clin Microbiol Infect Oxford, UK

31. Bavari S, Ulrich RG, LeClaire RD (1999) Cross-reactive antibodies prevent the lethal effects of Staphylococcus aureus superantigens. J Infect Dis 180(4):1365-1369

32. Fraser JD, Proft $T$ (2008) The bacterial superantigen and superantigen-like proteins. Immunol Rev 225:226-243

33. Zaluga E (1998) Skin reactions to antigens of propionibacterium acnes in patients with acne vulgaris treated with autovaccine. Ann Acad Med Stetin 44:65-85

34. Rusch V, Ottendorfer D, Zimmermann K (2001) Results of an open, non-placebo controlled pilot study investigating the immunomodulatory potential of autovaccine. Arzneimittelforschung 51(8):690 697

35. McLoughlin RM, Lee JC, Kasper DL, Tzianabos AO (2008) IFNgamma regulated chemokine production determines the outcome of Staphylococcus aureus infection. J Immunol 181(2):1323-1332

36. McLoughlin RM, Solinga RM, Rich J, Zaleski KJ, Cocchiaro JL, Risley A, Tzianabos AO, Lee JC (2006) CD4+ T cells and CXC chemokines modulate the pathogenesis of Staphylococcus aureus wound infections. Proc Natl Acad Sci USA 103(27):10408-10413

37. Xia G, Kohler T, Peschel A (2009) The wall teichoic acid and lipoteichoic acid polymers of Staphylococcus aureus. Int J Med Microbiol 